\title{
DECAY OF TEMPERATURE FLUCTUATIONS IN MHD TURBULENT FLOW IN PRESENCE OF DUST PARTICLES BEFORE THE FINAL PERIOD
}

\author{
M. L. RAHMAN \\ Department of Mathematics, Rajshahi University, Rajshahi-6205, Bangladesh
}

(Received revised March 14, 2007)

\section{ABSTRACT}

This paper reports the decay of temperature fluctuation in homogeneous MHD turbulence in the presence of dust particles before the final period. We have considered the two and three point correlation equations and solved them after neglecting the fourth order correlations in comparison with the second and third order correlations. Finally, the energy decay law for temperature fluctuation of MHD turbulence in the presence of dust particles at times before the final period is obtained.

\section{INTRODUCTION}

Saffman ${ }^{(1)}$ observed the effect of dust particles on the stability of the laminar flow of an incompressible fluid with constant mass concentration of dust particles and derived an equation which described the motion of a fluid containing small dust particles. It is of great interest of the behavior of dust particles in turbulent flow to many branches of science and technology, particularly if there is a substantial difference in density between the particles and the fluid. This behavior depends on the concentration and size of the particles with respect to the scale of turbulent flow. Deissler ${ }^{(2)}$ developed a theory "Decay of homogeneous turbulence for times before the final period”. He considered two and three point correlation equations neglecting fourth and higher order correlation terms. Using Deissler's theory, Kumar and Patel ${ }^{(3)}$ studied the " First order reactants in homogeneous turbulent flow before the final period" for the case of multipoint and single time correlation. Corrsin ${ }^{(4,5)}$ has already made an analytical attempt on the problem of turbulent temperature fluctuations using the approaches employed in the statistical theory of turbulence. Loeffler and Deissler ${ }^{(6)}$ studied the decay of temperature fluctuation in homogeneous turbulence before the final period. In their approach they considered the two and three point correlation equations and solved these equations after neglecting the fourth and higher order correlation terms. Following Deissler's approach, Sarker and Rahman $^{(7)}$ also studied the decay of temperature fluctuations in MHD turbulence before the final period. In this problem, we studied the decay of temperature fluctuation in MHD turbulence before the final period in the presence of dust particles as an extension of the work of Sarker and Rahman. ${ }^{(7)}$ Finally, the energy decay law for temperature field fluctuation of dusty fluid MHD turbulence before the final period is obtained.

\section{Two point correlation and spectral equations}


The induction equation of a magnetic field at the point $\mathrm{p}$ is

$\frac{\partial h_{i}}{\partial t}+u_{k} \frac{\partial h_{i}}{\partial x_{k}}-h_{k} \frac{\partial u_{i}}{\partial x_{k}}=\left(\frac{v}{p_{M}}\right) \frac{\partial^{2} h_{i}}{\partial x_{k} \partial x_{k}}$

and the energy equation at the point $p^{\prime}$ is

$\frac{\partial T_{J}^{\prime}}{\partial t}+u_{k}^{\prime} \frac{\partial T_{j}^{\prime}}{\partial x_{k}^{\prime}}=\left(\frac{v}{p_{r}}\right) \frac{\partial^{2} T_{j}^{\prime}}{\partial x_{k}^{\prime} \partial x_{k}^{\prime}}$

where

$u_{k}(\mathrm{x}, \mathrm{t})=$ component of turbulent velocity,

$h_{i}(x, t)=$ component of magnetic field,

$\rho_{M}=\frac{v}{\lambda}=$ magnetic Prandtl number,

$\rho_{r}=\frac{v}{\gamma}=$ Prandtl number,

$v=$ kinematics viscosity,

$\lambda=(4 \pi \mu \sigma)^{-1}=$ magnetic diffusivity,

$\gamma=\frac{k}{\rho c_{p}}=$ thermal diffusivity,

$c_{p}=$ heat capacity at constant pressure,

$T_{j}^{\prime}=$ component of temperature fluctuation,

$x_{k}=$ space co-ordinate.

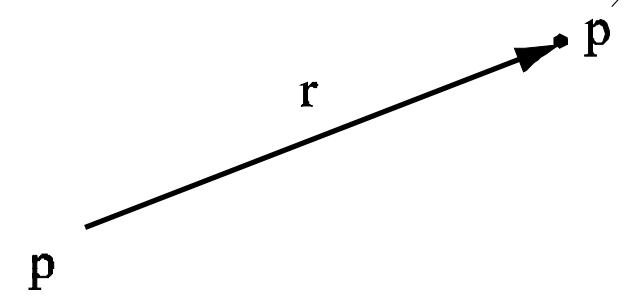

Fig.1. Vector configuration for two point correlation equations.

Multiplying equation (2.1) by $T_{j}{ }^{\prime}$ and (2.2) by $h_{i}$, adding and taking ensemble average, we get 


$$
\begin{aligned}
& \frac{\partial}{\partial t}\left(\overline{h_{i} T_{j}^{\prime}}\right)+\frac{\partial}{\partial x_{k}}\left(\overline{u_{k} h_{i} T_{j}^{\prime}}\right)+\frac{\partial}{\partial x_{k}^{\prime}} \overline{\left(\overline{\left.u_{k}^{\prime} h_{i} h_{j}^{\prime}\right)}-\frac{\partial}{\partial x_{k}}\left(\overline{u_{i} h_{k} T_{j}^{\prime}}\right)\right.} \\
& \left.\left.=v\left[\frac{1}{p_{M}} \frac{\partial^{2}}{\partial x_{k} \partial x_{k}} \overline{\left(h_{i} T_{j}^{\prime}\right.}\right)+\frac{1}{p_{r}} \frac{\partial^{2}}{\partial x_{k}^{\prime} \partial x_{k}^{\prime}} \overline{\left(h_{i} T_{j}^{\prime}\right.}\right)\right]
\end{aligned}
$$

The continuity equation is

$\frac{\partial u_{k}}{\partial x_{k}}=\frac{\partial u_{k}^{\prime}}{\partial x_{k}^{\prime}}=0$.

Substituting equation (2.4) into equation (2.3) yields

$$
\begin{aligned}
& \frac{\partial}{\partial t}\left(\overline{h_{i} T_{j}^{\prime}}\right)+\frac{\partial}{\partial x_{k}}\left(\overline{u_{k} h_{i} T_{j}^{\prime}}\right)+\frac{\partial}{\partial x_{k}^{\prime}}\left(\overline{u_{k}^{\prime} h_{i} T_{j}^{\prime}}\right)-\frac{\partial}{\partial{ }_{k}}\left(\overline{u_{i} h_{k} T_{j}^{\prime}}\right) \\
& =v\left[\frac{1}{P_{M}} \frac{\partial^{2}\left(\overline{h_{i} T_{j}^{\prime}}\right.}{\partial x_{k} \partial x_{k}}+\frac{1}{p_{r}} \frac{\partial^{2}\left(\overline{h_{i} T_{j}^{\prime}}\right)}{\partial x_{k}^{\prime} \partial x_{k}^{\prime}}\right]
\end{aligned}
$$

Using the transformations

$\frac{\partial}{\partial r_{k}}=-\frac{\partial}{\partial x_{k}}=\frac{\partial}{\partial x_{k}^{\prime}}$

and the relation (cf. Chandra Sekhar ${ }^{(8)}$ )

$\overline{u_{k} h_{i} T_{j}^{\prime}}=\overline{h_{i} u_{k}^{\prime} T_{j}^{\prime}}$ in equation (2.5), we get

$\left.\left.\left.\frac{\partial}{\partial t} \overline{\left(h_{i} T_{j}^{\prime}\right.}\right)+2 \frac{\partial}{\partial r_{k}} \overline{\left(u_{k}^{\prime} h_{i} T_{j}^{\prime}\right.}\right)+\frac{\partial}{\partial r_{k}}\left(\overline{u_{i} h_{k} T_{j}^{\prime}}\right)=v\left(\frac{1}{P_{M}}+\frac{1}{P_{r}}\right) \frac{\partial^{2}}{\partial r_{k} \partial r_{k}} \overline{\left(h_{i} T_{j}^{\prime}\right.}\right)$

Now, we write this equation in spectral form by use of the three dimensional Fourier transforms

$\overline{h_{i} T_{j}^{\prime}(r)}=\int_{-\infty}^{\infty} \overline{l_{i} \tau_{j}^{\prime}(k)} \exp (i k . r) d k$

$\overline{u_{i} h_{k} T_{j}^{\prime}}=\int_{-\infty}^{\infty} \overline{\phi_{i} l_{k} \tau_{j}^{\prime}(k)} \exp (i k . r) d k$

Interchanging the subscripts $\mathrm{i}$ and $\mathrm{j}$ and then interchanging the points $\mathrm{p}$ and $p^{\prime}$, we have $\overline{u_{k}^{\prime} h_{i} T_{j}^{\prime}(r)}=\overline{u_{k} h_{i} h_{j}^{\prime}(-r)}=\int_{-\infty}^{\infty} \overline{\phi_{k} l_{i} \tau_{j}^{\prime}}(-k) \cdot \exp (i k . r) d k$.

Putting (2.7), (2.8) and (2.9) into equation (2.6), we get

$\left.\frac{\partial}{\partial t} \overline{l_{i} \tau_{j}^{\prime}(k)}+i k_{k}\left[2 \overline{\phi_{k} l_{i} \tau_{j}(-k)}+\overline{\phi_{i} l_{k} \tau_{j}^{\prime}(k)}\right]=-v\left[\frac{1}{P_{M}}+\frac{1}{P_{r}}\right) K^{2} \overline{l_{i} \tau_{j}(K)}\right]$

The tensor equation (2.10) becomes a scalar equation by contraction of the indices $\mathrm{i}$ and $\mathrm{j}$

$\frac{\partial}{\partial t}\left(l_{i} \tau_{i}(k)+i k_{k}\left[2 \overline{\phi_{k} l_{i} \tau_{i}(-k)}+\overline{\phi_{i} l_{k} \tau_{i}^{\prime}}\right]=-v\left[\left(\frac{1}{p_{M}}+\frac{1}{p_{r}}\right) k^{2} \overline{l_{i} \tau_{i}^{\prime}(k)}\right]\right.$.

\section{Three point correlation and equations.}


The momentum equation of MHD turbulence in the presence of dust particles at the point $\mathrm{p}$, the induction equation at the point $\mathrm{p}^{\prime}$ and the energy equation at $\mathrm{p}$ " as

$$
\begin{aligned}
& \frac{\partial u_{i}}{\partial t}+u_{k} \frac{\partial u_{i}}{\partial x_{k}}-h_{k} \frac{\partial h_{i}}{\partial x_{k}}=-\frac{\partial W}{\partial x_{i}}+v \frac{\partial^{2} u_{i}}{\partial x_{k} \partial x_{k}}+\frac{K N}{\rho}\left(v_{i}-u_{i}\right) . \\
& \frac{\partial h_{i}^{\prime}}{\partial t}+u_{k}^{\prime} \frac{\partial h_{i}^{\prime}}{\partial x_{k}^{\prime}}-h_{k}^{\prime} \frac{\partial u_{i}^{\prime}}{\partial x_{k}^{\prime}}=\frac{v}{p_{M}} \frac{\partial^{2} h_{i}^{\prime}}{\partial x_{k}^{\prime} \partial x_{k}^{\prime}}
\end{aligned}
$$

and

$\frac{\partial T_{j}^{\prime \prime}}{\partial t}+u_{k} \frac{\partial T_{j}^{\prime \prime}}{\partial x^{\prime \prime}}=\left(\frac{v}{p_{r}}\right) \frac{\partial^{2} T_{j}^{\prime \prime}}{\partial x_{k}^{\prime \prime} \partial x_{k}^{\prime \prime}}$

where,

$W=\frac{P}{\rho}+\frac{1}{2}|\bar{h}|^{2}=$ total MHD pressure,

$\mathrm{P}(\mathrm{x}, \mathrm{t})=$ hydrodynamic pressure,

$\rho=$ fluid density,

$\mathrm{K}=$ stock registance,

$\mathrm{N}=$ number density of dust particles,

$v_{i}=$ component of the fluctuating velocity of dust particles.

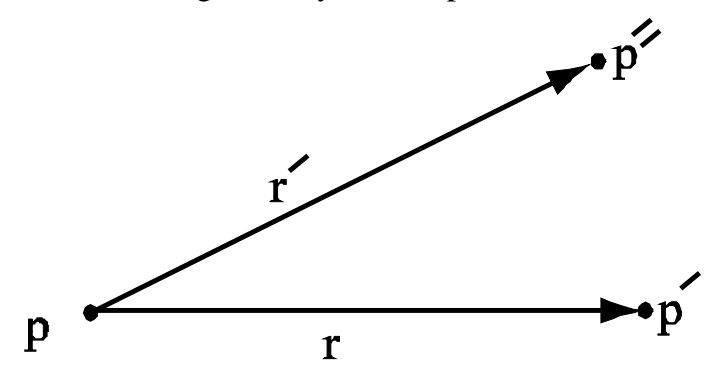

Fig.2. Vector configuration for three point correlation equations.

Multiplying the equation (3.1) by $h_{i} T_{j}^{\prime \prime}$, (3.2) by $u_{i} T_{j}^{\prime \prime}$, and (3.3) by $u_{i} h_{i}^{\prime}$, adding three equations and taking space or time averages, we obtain

$$
\begin{aligned}
& \left.\left.\left.\left.\frac{\partial}{\partial t} \overline{\left(u_{i} h_{j}^{\prime \prime} T_{j}^{\prime \prime}\right.}\right)+\frac{\partial}{\partial x_{k}} \overline{\left(u_{i} u_{k} h_{i}^{\prime} T_{j}^{\prime \prime}\right.}\right)-\frac{\partial}{\partial x_{k}} \overline{\left(h_{i} h_{k} h_{i}^{\prime} T_{j}^{\prime \prime}\right.}\right)+\frac{\partial}{\partial x_{k}^{\prime}} \overline{\left(u_{i} u_{k}^{\prime} h_{i}^{\prime \prime} T_{j}^{\prime \prime}\right.}\right)
\end{aligned}
$$

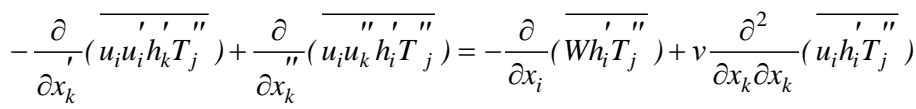

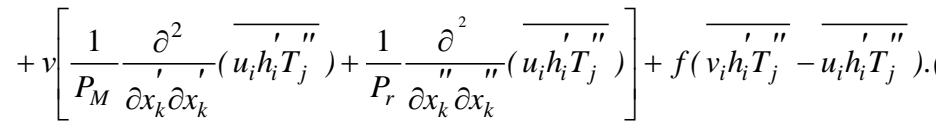

where $f=\frac{K N}{\rho}$ has the dimension of the frequency. Substituting the relations 
$\frac{\partial}{\partial x_{k}^{\prime}}=\frac{\partial}{\partial r_{k}}, \frac{\partial}{\partial x_{k}^{\prime \prime}}=\frac{\partial}{\partial r_{k}^{\prime}}$ and $\frac{\partial}{\partial x_{k}}=-\left(\frac{\partial}{\partial r_{k}}+\frac{\partial}{\partial r_{k}^{\prime}}\right)$ into equation (3.4), we get

$\frac{\partial}{\partial t}\left(\overline{\left(u_{i} h_{i}^{\prime} T_{j}^{\prime \prime}\right.}\right)-v\left[\left(1+\frac{1}{p_{M}}\right) \frac{\partial^{2}}{\partial r_{k} \partial r_{k}}\left(\overline{u_{i} h_{i}^{\prime \prime} T_{j}^{\prime \prime}}\right)+\left(1+\frac{1}{p_{r}}\right) \frac{\partial^{2}}{\partial r_{k}^{\prime} \partial r_{k}^{\prime}} \overline{\left(u_{i} h_{i}^{\prime \prime} T_{j}^{\prime \prime}\right.}\right)$

$\left.\left.\left.\left.+2 \frac{\partial^{2}}{\partial r_{k} \partial r_{k}^{\prime}} \overline{\left(u_{i} h_{i}^{\prime \prime} T_{j}^{\prime \prime}\right.}\right)\right]=\frac{\partial}{\partial r_{k}} \overline{\left(u_{i} u_{k} h_{i}^{\prime \prime} T_{j}^{\prime \prime}\right.}\right)+\frac{\partial}{\partial r_{k}^{\prime}}\left(\overline{u_{i} u_{k} h_{i}^{\prime} T_{j}^{\prime \prime}}\right)-\frac{\partial}{\partial r_{k}} \overline{\left(h_{i} h_{k} h_{i}^{\prime} T_{j}^{\prime \prime}\right.}\right)$

$-\frac{\partial}{\partial r_{k}}\left(\overline{\left(h_{i} h_{k} h_{i}^{\prime} T_{j}^{\prime \prime}\right.}\right)-\frac{\partial}{\partial r_{k}^{\prime}}\left(\overline{h_{i} h_{k} h_{i}^{\prime} T_{j}^{\prime \prime}}\right)-\frac{\partial}{\partial r_{k}}\left(\overline{u_{i} u_{k}^{\prime} h_{i}^{\prime} T_{j}^{\prime \prime}}\right)+\frac{\partial}{\partial r_{k}}\left(\left(\overline{u_{i} u_{i}^{\prime} h_{k}^{\prime} T_{j}^{\prime \prime}}\right) \mathrm{s}\right.$

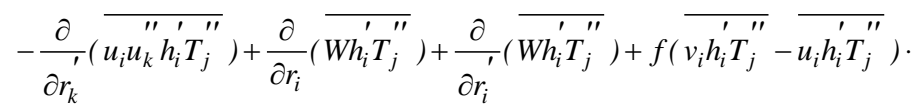

Now, we write equation (3.5) in spectral form in order to reduce it to an ordinary differential equation and because of the physical significance of spectral quantities. For this, we use six dimensional Fourier transforms:

$$
\begin{aligned}
& \overline{u_{i} h_{i}^{\prime}(r) T_{j}^{\prime \prime}\left(r^{\prime}\right)}=\int_{-\infty}^{\infty} \int_{-\infty}^{\infty} \overline{\phi_{i} \beta_{i}^{\prime}(k) \theta_{j}^{\prime \prime}\left(k^{\prime \prime}\right)} \cdot \exp \left[i\left(k \cdot r+k^{\prime} . r^{\prime}\right)\right] d k d k^{\prime}, \\
& \overline{u_{i} u_{i}^{\prime}(r) h_{k}^{\prime}(r) T_{j}^{\prime \prime}\left(r^{\prime}\right)}=\int_{-\infty}^{\infty} \int_{-\infty}^{\infty} \overline{\phi_{i} \phi_{i}^{\prime}(k) \beta_{k}^{\prime}(k)} \theta_{j}^{\prime \prime}\left(k^{\prime}\right) \cdot \exp \left[i\left(k \cdot r+k^{\prime} \cdot r^{\prime}\right)\right] d k d k^{\prime} \text {, }
\end{aligned}
$$

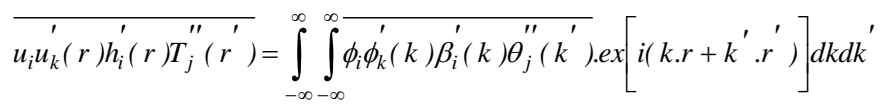

$$
\begin{aligned}
& \overline{u_{i} u_{k} h_{i}^{\prime}(r) T_{j}^{\prime \prime}\left(r^{\prime}\right)}=\int_{-\infty}^{\infty} \int_{-\infty}^{\infty} \overline{\phi_{i} \phi_{k} \beta_{i}^{\prime}(k) \theta_{j}^{\prime \prime}\left(k^{\prime}\right)} \cdot \exp \left[i\left(k \cdot r+k^{\prime} \cdot r^{\prime}\right)\right] d k d k^{\prime} \\
& \left.\overline{h_{i} h_{k} h_{i}^{\prime}(r) T_{j}^{\prime \prime}\left(r^{\prime}\right)}=\int_{-\infty-\infty}^{\infty} \int_{\beta_{i} \beta_{k} \beta_{i}^{\prime} \theta_{j}^{\prime \prime}\left(k^{\prime}\right)}\right) \cdot \exp \left[i\left(k \cdot r+k^{\prime} \cdot r^{\prime}\right)\right] d k d k^{\prime}
\end{aligned}
$$

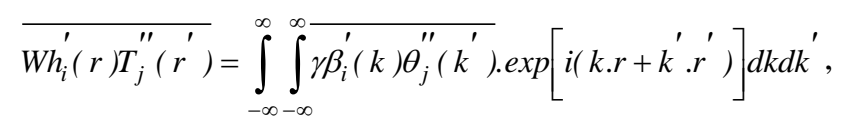

Interchanging of points $p^{\prime}$ and $p^{\prime \prime}$ along with the indices $\mathrm{i}$ and $\mathrm{j}$, result in the relations

$$
\overline{u_{i} u_{k}^{\prime \prime} h_{i}^{\prime} T_{j}^{\prime \prime}}=\overline{u_{i} u_{k}^{\prime} h_{i}^{\prime} T_{j}^{\prime \prime}}
$$

By use of these facts and relations (3.6) - (3.12), one can write equation (3.5) in the form $\frac{\partial}{\partial t} \overline{\phi_{i} \beta_{i}^{\prime} \theta_{j}^{\prime \prime}}+v\left[\left(1+\frac{1}{P_{M}}\right) k^{2}+\left(1+\frac{1}{p_{r}}\right) k^{\prime 2}+2 k_{k} k_{k}^{\prime}\right] \overline{\phi_{i} \beta_{i}^{\prime} \theta_{j}^{\prime \prime}}$ 


$$
\begin{aligned}
& =i\left(k_{k}+k_{k}^{\prime}\right) \overline{\phi_{i} \phi_{k} \beta_{i}^{\prime} \theta_{j}^{\prime \prime}}-i\left(k_{k}+k_{k}^{\prime}\right) \overline{\beta_{i} \beta_{k} \beta_{i}^{\prime} \theta_{j}^{\prime \prime}}-i\left(k_{k}+k_{k}^{\prime}\right) \overline{\phi_{i} \phi_{k}^{\prime} \beta_{i}^{\prime} \theta_{j}^{\prime \prime}} \\
& \left.+i\left(k_{k}+k_{k}^{\prime}\right) \overline{\phi_{i} \phi_{i}^{\prime} \beta_{k}^{\prime} \theta_{j}^{\prime \prime}}+i\left(k_{i}+k_{i}^{\prime}\right) \overline{\beta_{i}^{\prime} \theta_{j}^{\prime \prime}}+f \overline{\left(\mu_{i} \beta_{i}^{\prime} \theta_{j}^{\prime \prime}\right.}-\overline{\phi_{i} \beta_{i}^{\prime} \theta_{j}^{\prime \prime}}\right)
\end{aligned}
$$

The tensor equation (3.13) can be converted to scalar equation by contraction of the subscripts $i$ and $j$

$$
\begin{aligned}
& \frac{\partial}{\partial t} \overline{\phi_{i} \beta_{i}^{\prime} \theta_{j}^{\prime \prime}}+v\left[\left(1+\frac{1}{P_{M}}\right) k^{2}+\left(1+\frac{1}{P_{r}}\right) k^{\prime 2}+2 k_{k} k_{k}^{\prime}\right] \overline{\phi_{i} \beta_{i}^{\prime} \theta_{j}^{\prime \prime}}=i\left(k_{k}+k_{k}^{\prime}\right) \overline{\phi_{i} \phi_{k} \beta_{i}^{\prime} \theta_{i}^{\prime \prime}} \\
& -i\left(k_{k}+k_{k}^{\prime}\right) \overline{\beta_{i} \beta_{k} \beta_{i}^{\prime} \theta_{i}^{\prime \prime}}-i\left(k_{k}+k_{k}^{\prime}\right) \overline{\phi_{i} \phi_{k} \beta_{i}^{\prime} \theta_{i}^{\prime \prime}}+i k_{k} \overline{\phi_{i} \phi_{i}^{\prime} \beta_{k}^{\prime} \theta_{i}^{\prime \prime}} \\
& +i\left(k_{k}+k_{k}^{\prime}\right) \overline{\beta_{i}^{\prime} \theta_{j}^{\prime \prime}}+f\left(\overline{\mu_{i} \beta_{i}^{\prime} \theta_{j}^{\prime \prime}}-\overline{\phi_{i} \beta_{i}^{\prime} \theta_{j}^{\prime \prime}}\right)
\end{aligned}
$$

If we take the derivative with respect to $x_{i}$ of the momentum equation (3.1) for the point $\mathrm{p}$, we obtain

- $-\frac{\partial^{2} W}{\partial x_{i} \partial x_{i}}=\frac{\partial^{2}}{\partial x_{i} \partial x_{k}}\left(u_{i} u_{k}-h_{i} h_{k}\right)-\frac{\partial}{\partial x_{i}} f\left(v_{i}-u_{i}\right)$

Multiplying equation (3.15) by $h_{i}^{\prime} T_{j}^{\prime \prime}$, taking averages and writing the equation in terms of the independent variables $r$ and $r^{\prime}$

$$
\begin{aligned}
& -\left[\frac{\partial^{2}}{\partial r_{i} \partial r_{i}}+\frac{\partial^{2}}{\partial r_{i}^{\prime} \partial r_{i}^{\prime}}+2 \frac{\partial^{2}}{\partial r_{i} \partial r_{i}^{\prime}}\right] \overline{w h_{i}^{\prime} T_{j}^{\prime \prime}} \\
& \left.=\left[\frac{\partial^{2}}{\partial r_{i} \partial r_{k}}+\frac{\partial^{2}}{\partial r_{i}^{\prime} \partial r_{k}}+\frac{\partial^{2}}{\partial r_{i} \partial r_{k}^{\prime}}+\frac{\partial^{2}}{\partial r_{i}^{\prime} \partial_{k}^{\prime}}\right] \overline{\left(u_{i} u_{k} h_{i}^{\prime} T_{j}^{\prime \prime}\right.}-\overline{h_{i} h_{k} h_{i}^{\prime} T_{j}^{\prime \prime}}\right) \\
& +f\left(\frac{\partial}{\partial r_{i}}+\frac{\partial}{\partial r_{i}^{\prime}}\right)\left(\overline{\left(v_{i}^{\prime} h_{i}^{\prime \prime} T_{j}^{\prime \prime}\right.}-\frac{u_{i}^{\prime} h_{i}^{\prime \prime} T_{j}^{\prime \prime}}{}\right) .
\end{aligned}
$$

Taking the Fourier transforms of equation (3.16), we get

$-\overline{-\gamma \beta_{i}^{\prime} \theta_{j}^{\prime \prime}}=\left[\left(k_{i} k_{k}+k_{i}^{\prime} k_{k}+k_{i} k_{k}^{\prime}+k_{i}^{\prime} k_{k}^{\prime}\left(\overline{\phi_{i} \phi_{k} \beta_{i}^{\prime} \theta_{j}^{\prime \prime}}-\overline{\beta_{i} \beta_{k} \beta_{i}^{\prime} \theta_{j}^{\prime \prime}}\right)\right.\right.$

$\left.\left.-i f\left(k_{i}+k_{i}^{\prime}\right) \overline{\left(\mu \beta_{i}^{\prime} \theta_{j}^{\prime \prime}\right.}-\overline{\phi_{i} \beta_{i}^{\prime} \theta_{j}^{\prime \prime}}\right)\right] /\left(k^{2}+k^{\prime 2}+2 k_{i} k_{i}^{\prime}\right)$.

Equation (3.1) can be used to eliminate $\overline{\gamma \beta_{i}^{\prime} \theta_{j}^{\prime \prime}}$ from equation (3.13).

\section{Solution for times before the final period}

To study the decay of MHD dusty fluid turbulence for times before the final period, the three point correlations are considered and the quadruple correlations are neglected. If this is happened then equation (3.17) shows that the term $\overline{\gamma \beta_{i}^{\prime} \theta_{j}^{\prime \prime}}$ associated with the pressure correlations, should also be neglected. Thus we have from the equation (3.14)

$\frac{\partial}{\partial t} \overline{\phi_{i} \beta_{i}^{\prime} \theta_{j}^{\prime \prime}}+v\left[\left(1+\frac{1}{p_{M}}\right) k^{2}+\left(1+\frac{1}{p_{r}}\right) k^{\prime 2}+2 k k^{\prime}-R f\right] \overline{\phi_{i} \beta_{i}^{\prime} \theta_{i}^{\prime \prime}}=0$. 
where

$R=\left\{\frac{\left(k_{i}+k_{i}^{\prime}\right)^{2}}{k^{2}+k^{\prime^{2}}+2 k_{i} k_{i}^{\prime}}-1\right\}(s-1)$

and $\quad \mu \beta_{i}^{\prime} \theta_{j}^{\prime \prime}=s \phi_{i} \beta_{i}^{\prime} \theta_{j}^{\prime \prime}$, also R and s are arbitrary constants.

Integrating the equation (4.1) between $t_{o}$ and t with inner multiplication by $k_{k}$ gives

$\overline{k_{k} \phi_{i} \beta_{i}^{\prime} \theta_{i}^{\prime \prime}}=k_{k}\left[\overline{\phi_{i} \beta_{i}^{\prime} \theta_{i}^{\prime \prime}}\right]_{0} \cdot \exp \left[\left\{-v\left[\left(1+\frac{1}{p_{M}}\right) k^{2}+\left(1+\frac{1}{p r}\right) k^{\prime^{2}}+2 k k^{\prime} \cos \theta\right]+R f\right\}\left(t-t_{0}\right)\right]$

where $\theta$ is the angle between $\mathrm{k}$ and $k^{\prime}$. Now, by letting $r^{\prime}=0$ in equation (3.6) and comparing with the equation (2.8) and (2.9), we have

$\overline{\phi_{i} l_{k} \tau_{i}^{\prime}(k)}=\int_{-\infty}^{\infty} \overline{\phi_{i} \beta_{i}^{\prime \prime} \theta_{i}^{\prime \prime}} d k^{\prime}$

and

$\overline{\phi_{i} l_{i} \tau_{i}^{\prime}(-k)}=\int_{-\infty}^{\infty} \overline{\phi_{k} \beta_{i}^{\prime}(-k) \theta_{i}^{\prime \prime}\left(-k^{\prime}\right)} d k^{\prime}$

Substituting equations (4.2), (4.3) and (4.4) in equation (2.9), we get

$\frac{\partial}{\partial t} \overline{l_{i} \tau_{i}^{\prime}(k)}+v\left(\frac{1}{p_{M}}+\frac{1}{p_{r}}\right) k^{2} \overline{l_{i} \tau_{i}^{\prime}(k)}=\int_{-\infty}^{\infty}\left[\overline{\phi_{i} \beta_{i}^{\prime} \theta_{i}^{\prime \prime}}+2 \overline{\phi_{k} \beta_{i}^{\prime}(-k) \theta_{i}^{\prime \prime}\left(-k^{\prime}\right)}\right]_{0}$

. $\exp \left[\left\{-v\left[\left(1+\frac{1}{p_{M}}\right) k^{2}+\left(1+\frac{1}{p r}\right) k^{\prime 2}+2 k k^{\prime} \cos \theta\right]+R f\right\}\left(t-t_{0}\right)\right] d k^{\prime}$

Now, $d k^{\prime}=d k_{1}^{\prime} d k_{2}^{\prime} d k_{3}^{\prime}$ can be expressed intersms of $k^{\prime}$ and $\theta^{(3)}$ as

$d k^{\prime}=-2 \pi k^{\prime 2} d(\cos \theta) d k^{\prime}$

Substituting equation (4.6) to equation (4.5) to give

$$
\begin{aligned}
& \left.\frac{\partial}{\partial t} \overline{l_{i} \tau_{i}^{\prime}(k)}+v\left(\frac{1}{p_{M}}+\frac{1}{p_{r}}\right) k^{2} \overline{l_{i} \tau_{i}^{\prime}(k)}=-\int_{0}^{\infty} 2 \pi i k_{k} \overline{\phi_{i} \beta_{i}^{\prime} \theta_{i}^{\prime \prime}}+2 \overline{\phi_{k} \beta_{i}^{\prime}(-k) \theta_{i}^{\prime \prime}\left(-k^{\prime}\right)}\right]_{0} k^{\prime 2} \\
& \left.\cdot \int_{-1}^{1} \exp \left[\left\{-v\left[\left(1+\frac{1}{p_{M}}\right) k^{2}+\left(1+\frac{1}{p r}\right) k^{\prime 2}+2 k k^{\prime} \cos \theta\right]+R f\right\}\left(t-t_{0}\right)\right] d(\cos \theta)\right] d k^{\prime} .
\end{aligned}
$$

In order to find the solution completely and following Loeffler and Deissler ${ }^{(6)}$, we assume that

$i k_{k}\left[\overline{\phi_{i} \beta_{i}^{\prime} \theta_{i}^{\prime \prime}}+2 \overline{\phi_{k} \beta_{i}^{\prime}(-k) \theta_{i}^{\prime \prime}\left(-k^{\prime}\right)}\right]_{0}=-\frac{\xi_{o}}{(2 \pi)^{2}}\left(k^{2} k^{{ }^{4}}-k^{4} k^{\prime 2}\right)$

where $\xi_{0}$ is a constant depending on the initial conditions.

Putting (4.8) in equation (4.7) and completing the integration with respect to $\cos \theta$, we get 


$$
\begin{aligned}
& \frac{\partial}{\partial t} \overline{\left(2 \pi l_{i} \tau_{i}^{\prime}(k)\right.}+v\left(\frac{1}{p_{M}}+\frac{1}{p_{r}}\right) k^{2}\left(2 \pi \bar{l}_{i} \tau_{i}^{\prime}(k)\right)=-\frac{\xi_{0}}{2 v\left(t-t_{0}\right)} \int_{0}^{\infty}\left(k k^{\prime 5}-k^{3} k^{\prime 3}\right) \\
& {\left[\exp \left[\left\{-v\left[\left(1+\frac{1}{p_{M}}\right) k^{2}+\left(1+\frac{1}{p r}\right) k^{\prime 2}-2 k k^{\prime}\right]+R f\right\}\left(t-t_{0}\right)\right]\right.} \\
& \text { exp } \left.\left[\left\{-v\left[\left(1+\frac{1}{p_{M}}\right) k^{2}+\left(1+\frac{1}{p r}\right) k^{\prime 2}+2 k k^{\prime}\right]+R f\right\}\left(t-t_{0}\right)\right]\right] d k^{\prime}
\end{aligned}
$$

Multiplying both sides by $k^{2}$, we have

$\frac{\partial Q}{\partial t}+v\left(\frac{1}{p_{M}}+\frac{1}{p_{r}}\right) k^{2} Q=F$

where

$Q=2 \pi k^{2} \overline{l_{i} \tau_{i}^{\prime}(k)}$

is the magnetic energy spectrum function and $\mathrm{F}$ is the energy transfer term given by

$$
\begin{aligned}
& \mathrm{F}=-\frac{\xi_{0}}{v\left(\mathrm{t}-\mathrm{t}_{0}\right)} \int_{0}^{\infty}\left(\mathrm{k}^{3} \mathrm{k}^{15}-\mathrm{k}^{5} \mathrm{k}^{\prime 3}\right)\left[\operatorname { e x p } \left[\left\{-v\left[\left(1+\frac{1}{\mathrm{p}_{\mathrm{M}}}\right) \mathrm{k}^{2}+\left(1+\frac{1}{\mathrm{p}_{\mathrm{r}}}\right) \mathrm{k}^{\prime 2}\right.\right.\right.\right. \\
& \left.\left.\left.\left.-2 k k^{\prime}\right]+R f\right\}\left(t-t_{o}\right)\right]-\exp \left[\left\{-v\left[\left(1+\frac{1}{p_{M}}\right) k^{2}+\left(1+\frac{1}{p_{r}}\right) k^{\prime 2}+2 k k^{\prime}\right]+R f\right\}\left(t-t_{o}\right)\right]\right] d k^{\prime} . .
\end{aligned}
$$

Integrating equation (4.12) with respect to $k^{\prime}$, we obtain

$$
\begin{aligned}
& F=-\frac{\sqrt{\pi} \xi_{0} p_{r}^{\frac{5}{2}}}{2 v^{\frac{3}{2}}\left(t-t_{o}\right)^{\frac{3}{2}}\left(1+p_{r}\right)^{\frac{5}{2}}} \exp \left[\left\{-v\left(1+\frac{1}{p_{M}}-\frac{p_{r}}{1+p_{r}}\right) k^{2}+R f\right\}\left(t-t_{0}\right)\right] \\
& {\left[\frac{15 p_{r} k^{4}}{4 v^{2}\left(t-t_{0}\right)^{2}\left(1+p_{r}\right)}+\left\{\frac{5 p_{r}{ }^{2}}{\left(1+p_{r}\right)^{2}}-\frac{3}{2}\right\} \frac{k^{6}}{v\left(t-t_{0}\right)}+\left\{\frac{p_{r}{ }^{3}}{\left(1+p_{r}\right)^{3}}-\frac{p_{r}}{\left(1+p_{r}\right)}\right\} k^{8}\right]}
\end{aligned}
$$

The series of equation (4.13) contains only even power of $\mathrm{k}$.

It is interesting to note that

$\int_{0}^{\infty} F \cdot d k=0$

This indicates that the condition of continuity and homogeneity are maintained.

The linear equation (4.10) can be solved to give

$$
\begin{aligned}
& Q=\exp \left[-\imath k^{2}\left(t-t_{0}\right)\left(\frac{1}{p_{M}}+\frac{1}{p_{r}}\right)\right] \int F \cdot \exp \left[\imath k^{2}\left(\frac{1}{p_{M}}+\frac{1}{p_{r}}\right)\left(t-t_{o}\right)\right] d t \\
& +C(k) \exp \left[-\imath k^{2}\left(\frac{1}{p_{M}}+\frac{1}{p_{r}}\right)\left(t-t_{o}\right)\right]
\end{aligned}
$$

where $C(k)=\frac{N_{0} k^{2}}{\pi}(4.16)$ is a constant of integration and can be obtained as by corrsin. ${ }^{(4)}$ Substituting the values of $\mathrm{F}$ from equation (4.13) in equation (4.15) and integrating with respect to $t$, we get $Q(k, t)=\frac{N_{0} k^{2}}{\pi} \exp \left[\left\{-v K^{2}\left(\frac{1}{p_{M}}+\frac{1}{p_{r}}\right)+R f\right\}\left(t-t_{0}\right)\right]+\frac{\sqrt{\pi} \xi_{0} p_{r}^{\frac{5}{2}}}{2 v^{\frac{3}{2}\left(1+p_{r}\right)^{\frac{7}{2}}}} \exp \left[\left\{-v^{2} \frac{\left(1+P_{r}+P_{M}\right)}{P_{M}\left(1+p_{r}\right)}+R f\right\}\left(t-t_{0}\right)\right]$ 
$\left[\frac{3 p_{r} k^{4}}{2 v^{2}\left(t-t_{0}\right)^{\frac{5}{2}}}+\frac{p_{r}\left(7 p_{r}-6\right) k^{6}}{3 v\left(1+p_{r}\right)\left(t-t_{0}\right)^{\frac{3}{2}}}-\frac{4\left(3 p_{r}{ }^{2}-2 p_{r}+3\right) k^{8}}{3\left(1+p_{r}\right)^{2}\left(t-t_{0}\right)^{\frac{1}{2}}}+\frac{8 \sqrt{v}}{3} \frac{\left(3 p_{r}{ }^{2}-2 p_{r}+3\right) k^{9}}{3\left(1+p_{r}\right)^{\frac{5}{2}} p_{r} \frac{1}{2}} N(\omega)\right]$

where $N(\omega)=\exp \left(-\omega^{2}\right) \int_{0}^{W} \exp \left(x^{2}\right) d x$ and

$\omega=k\left[\frac{v\left(t-t_{0}\right)}{p_{M}\left(1+p_{M}\right)}\right]^{\frac{1}{2}}$.

The function $F(\omega)$ has been calculated numerically and tabulated in. ${ }^{(6)}$ Let $\mathbf{r}=0$ in equation (2.7) and use is made of the definition of $\mathrm{Q}$ as given by equation(4.11), the result is

$\frac{\overline{T^{2}}}{2}=\frac{\overline{T_{i} T_{j}^{\prime}}}{2}=\int_{0}^{\infty} Q(k) d k$

Substituting equation (4.17) into (4.18) and after integration, one can obtain

$\frac{\overline{T^{2}}}{2}=\frac{N_{0} P_{M}{ }^{\frac{3}{2}} v^{\frac{-3}{2}}\left(t-t_{0}\right)^{-\frac{3}{2}}}{8 \sqrt{2 \pi}}+\exp \left\{R f\left(t-t_{0}\right)\right\} \xi_{0} S v^{-6}\left(t-t_{0}\right)^{-5}$

where

$S=\frac{\pi P_{M}{ }^{6}}{\left(1+P_{M}\right)\left(1+2 P_{M}\right)^{\frac{3}{2}}}\left[\frac{9}{16}+\frac{5 P_{M}\left(7 P_{M}-6\right)}{16\left(1+2 P_{M}\right)}-\frac{35 P_{M}\left(3 P_{M}{ }^{2}-2 P_{M}+3\right)}{8\left(1+2 P_{M}\right)^{2}}+\ldots . ..\right]$.

Thus, the decay law for magnetic energy fluctuation before the final period in the presence of dust particle may be written as

$\overline{T^{2}}=A\left(t-t_{0}\right)^{-\frac{3}{2}}+B\left(t-t_{0}\right)^{-5} \exp \left\{R f\left(t-t_{0}\right)\right\}$,

where, $\overline{T^{2}}$ is the mean square of the magnetic field fluctuation, $\mathrm{t}$ is the time,

$A=\frac{N_{0} P_{M}{ }^{\frac{3}{2}} v^{-\frac{3}{2}}}{4 \sqrt{2 \pi}}, \quad B=2 \xi_{0} Q v^{-6}$ and $\mathrm{t}_{0}$ are constants determined by the initial conditions.

For larger times, the last terms in the equation becomes negligible, leaving the $-3 / 2$ power decay law for the final period.

The results of the present study, obtained by neglecting the quadruple correlation's in the three points correlation equation, appear to represent the MHD turbulence for times before the final period. For clean fluid , i.e. in absence of dust particles we put $f=0$, the equation (4.20) becomes

$\overline{T^{2}}=A\left(t-t_{0}\right)^{-\frac{3}{2}}+B\left(t-t_{0}\right)^{-5}$

which was obtained earlier by Sarker and Rahman. ${ }^{(8)}$ For larger times, the last term in the equation becomes negligible, giving the $-3 / 2$ power decay law for the final period.

\section{REFERENCES}

1. P. G. SAfFman, “On the stability of laminar flow of a dusty gas”, J. Fluid Mech., 13, 120, 1962. 
2. R. G. DeISSLER, “On the decay of homogeneous turbulence before the final period”, Physics Flud 1, 111, 1958.

3. P. KumAR. AND S. R. PATEL, "First order reactant in homogeneous turbulent flow before the final period”, Physics, Fluids 17, 1362, 1974.

4. S. CORRSIN, J.Appl.Phys. 22, 469-473, 1951.

5. S. Corrsin, J. Aeronaut. Sci., 18, 417, 1951.

6. A. L. LoEfFler, AND R. G. Deissler, "Decay of temperature fluctuation in homogeneous turbulence before the final period”, Int . J. heat transfer 1, 312, 1961.

7. S. A. SARKER, AND M. L.. RAHMAN, "Decay of temperature fluctuations in MHD turbulence before the final period”, North Bengal University Review, India .9, 1 91-102, 1998.

8. Chandra Sekhar, S. Proc. R. Soc., London, A 204, 435, 1951.

Journal of Bangladesh Academy of Sciences, Vol. 32, No. 1, 61-70, 2008 